\title{
THA-SURFACES IN 3-DIMENSIONAL EUCLIDEAN SPACE
}

\author{
BENDEHIBA SENOUSSI $^{1, *}$, ABDELAZIZ BENNOUR ${ }^{2}$, KHALED BEDDANI $^{2}$ \\ ${ }^{1}$ Department of Mathematics, Ecole Normale Supérieure de Mostaganem, Algeria \\ ${ }^{2}$ Department of Mathematics, Abdelhamid Ibnbadis University, Algeria \\ *Corresponding author: se2014bendhiba@gmail.com
}

Received Dec. 12, 2020

\begin{abstract}
Aвstract. In this paper, we define THA-surfaces in the 3-dimensional Euclidean space $\mathbb{E}^{3}$ and completely classify minimal or flat THA-surfaces. We study the THA-surfaces in $\mathbb{E}^{3}$ under the condition $\Delta r_{i}=\lambda_{i} r_{i}$, where $\lambda_{i} \in \mathbb{R}$ and $\Delta$ denotes the Laplace operator. We obtain the complete classification for those ones.

2010 Mathematics Subject Classification. 53A10; 53C42.
\end{abstract}

Key words and phrases. THA-surfaces; finite type immersion; Laplacian operator.

\section{INTRODUCTION}

Let $\mathbb{E}^{3}$ be a three-dimensional Euclidean space. We recall some well-known formulas for the surfaces in $\mathbb{E}^{3}$. Let $r=r(u, v)$ be an isometric immersion of a surface $M^{2}$ in $\mathbb{E}^{3}$. The inner product on $\mathbb{E}^{3}$ is

$$
g(X, Y)=x_{1} y_{1}+x_{2} y_{2}+x_{3} y_{3},
$$

where $X=\left(x_{1}, x_{2}, x_{3}\right), Y=\left(y_{1}, y_{2}, y_{3}\right) \in \mathbb{R}^{3}$.

The Euclidean vector product $X \wedge Y$ of $X$ and $Y$ is defined as follows:

$$
X \wedge Y=\left(x_{2} y_{3}-x_{3} y_{2}, x_{3} y_{1}-x_{1} y_{3}, x_{1} y_{2}-x_{2} y_{1}\right) .
$$

The notion of finite type immersion of submanifolds of a Euclidean space has been widely used in classifying and characterizing well known Riemannian submanifolds [3]. An Euclidean submanifold is said to be of Chen finite type if its coordinate functions are a finite sum of eigenfunctions of its Laplacian $\Delta[3]$. B.-Y. Chen posed the problem of classifying the finite

DOI: 10.28924/APJM/8-10

(2021 Asia Pacific Journal of Mathematics 
type submanifolds in the 3-dimensional Euclidean space $\mathbb{E}^{3}$. These can be regarded as a generalization of minimal submanifolds.

The notion of finite type immersion has played an important role in classifying and characterizing the submanifolds in Euclidean space.

Since then the theory of submanifolds of finite type has been studied by many geometers.

A well known result due to Takahashi [16] states that minimal surfaces and spheres are the only surfaces in $\mathbb{E}^{3}$ satisfying the condition

$$
\Delta r=\lambda r, \lambda \in \mathbb{R}
$$

In [5] Ferrandez, Garay and Lucas proved that the surfaces of $\mathbb{E}^{3}$ satisfying

$$
\Delta H=A H, A \in \operatorname{Mat}(3,3)
$$

are either minimal, or an open piece of sphere or of a right circular cylinder.

In [4] F. Dillen, J. Pas and L. Verstraelen proved that the only surfaces in $\mathbb{E}^{3}$ satisfying

$$
\Delta r=A r+B, A \in \operatorname{Mat}(3,3), B \in \operatorname{Mat}(3,1),
$$

are the minimal surfaces, the spheres and the circular cylinders.

In [1], the authors classified the factorable surfaces in the three-dimensional Euclidean and Lorentzian spaces, whose component functions are eigenfunctions of their Laplace operator. The authors in [2] studied the translation surfaces in the 3-dimensional Euclidean and LorentzMinkowski space under the condition

$$
\Delta^{I I I} r_{i}=\mu_{i} r_{i}, \quad \mu_{i} \in \mathbb{R}
$$

where $\Delta^{I I I}$ denotes the Laplacian of the surface with respect to the third fundamental form III.

In this paper, we define THA-surfaces in the 3-dimensional Euclidean space $\mathbb{E}^{3}$ and completely classify minimal or flat THA-surfaces. We study the THA-surfaces in $\mathbb{E}^{3}$ under the condition $\Delta r_{i}=\lambda_{i} r_{i}$, where $\lambda_{i} \in \mathbb{R}$. We obtain the complete classification for those ones.

\section{Preliminaries}

A submanifold $M^{2}$ of a 3-dimensional Euclidean space $\mathbb{E}^{3}$ is said to be of finite type if each component of its position vector field $r$ can be written as a finite sum of eigenfunctions of the 
Laplacian $\Delta$ of $M^{2}$, that is, if

$$
r=r_{0}+\sum_{i=1}^{k} r_{i}
$$

where $r_{i}$ are $\mathbb{E}^{3}$-valued eigenfunctions of the Laplacian of $\left(M^{2}, r\right)[3]$ :

$$
\Delta r_{i}=\lambda_{i} r_{i}
$$

where $\lambda_{i} \in \mathbb{R}, i=1,2, . ., k$. If $\lambda_{i}$ are different, then $M^{2}$ is said to be of $k$-type.

The coefficients of the first fundamental form and the second fundamental form are

$$
\begin{aligned}
& E=g\left(r_{u}, r_{u}\right), F=g\left(r_{u}, r_{v}\right), G=g\left(r_{v}, r_{v}\right), \\
& L=g\left(r_{u u}, \mathbf{N}\right), M=g\left(r_{u v}, \mathbf{N}\right), N=g\left(r_{v v}, \mathbf{N}\right),
\end{aligned}
$$

where $r_{u}=\frac{\partial r}{\partial u}, r_{v}=\frac{\partial r}{\partial v}$ and $\mathbf{N}$ is the unit normal vector to $M^{2}$.

The Laplace-Beltrami operator of a smooth function $\varphi: M^{2} \rightarrow \mathbb{R},(u, v) \mapsto \varphi(u, v)$ with respect to the first fundamental form of the surface $M^{2}$ is the operator $\Delta$, defined in $[13,15]$ as follows:

$$
\Delta \varphi=\frac{-1}{\sqrt{\left|E G-F^{2}\right|}}\left[\frac{\partial}{\partial u}\left(\frac{G \varphi_{u}-F \varphi_{v}}{\sqrt{\left|E G-F^{2}\right|}}\right)+\frac{\partial}{\partial v}\left(\frac{E \varphi_{v}-F \varphi_{u}}{\sqrt{\left|E G-F^{2}\right|}}\right)\right] .
$$

The second differential parameter of Beltrami of a function $\varphi: M^{2} \rightarrow \mathbb{R},(u, v) \longmapsto \varphi(u, v)$ with respect to the second fundamental form of $M^{2}$ is the operator $\Delta^{I I}$ which is defined by $[13,15]$

$$
\Delta^{I I} \varphi=\frac{-1}{\sqrt{\left|L N-M^{2}\right|}}\left[\frac{\partial}{\partial u}\left(\frac{N \varphi_{u}-M \varphi_{v}}{\sqrt{\left|L N-M^{2}\right|}}\right)+\frac{\partial}{\partial v}\left(\frac{L \varphi_{v}-M \varphi_{u}}{\sqrt{\left|L N-M^{2}\right|}}\right)\right],
$$

where $L N-M^{2} \neq 0$ since the surface has no parabolic points.

The mean curvature $H$ and the Gaussian curvature $K_{G}$ are, respectively, defined by

$$
H=\frac{E N+G L-2 F M}{2\left(E G-F^{2}\right)} \text { and } K_{G}=\frac{L N-M^{2}}{E G-F^{2}} .
$$

\section{THA-surfaces IN $\mathbb{E}^{3}$}

Let $M^{2}$ be a 2-dimensional surface of the Euclidean 3-space $\mathbb{E}^{3}$. Using the standard coordinate system of $\mathbb{E}^{3}$ we denote the parametric representation of the surface $r(u, v)$ by

$$
r(u, v)=\left(r_{1}(u, v), r_{2}(u, v), r_{3}(u, v)\right)
$$

In $\mathbb{E}^{3}$, a surface is called a translation surface if it is given by an immersion 


$$
r: \Omega \subset \mathbb{R}^{2} \rightarrow \mathbb{R}^{3}:(u, v) \mapsto(u, v, f(u)+g(v)),
$$

where $f$ and $g$ are smooth functions on opens of $\mathbb{R}$. One of the famous examples of minimal surfaces in 3-dimensional Euclidean space $\mathbb{E}^{3}$ is a Scherk's minimal translation surface. In fact, Scherk showed in 1835 that except the planes, the only minimal translation surfaces are the surfaces given by

$$
r(u, v)=\left(u, v, \frac{1}{\lambda} \log \cos (\lambda v)-\frac{1}{\lambda} \log \cos (\lambda u)\right),
$$

where $\lambda$ is a nonzero constant. This surface is called a Scherk's minimal translation surface.

R. López [8] studied translation surfaces in the 3-dimensional hyperbolic space $\mathbb{H}^{3}$ and classified minimal translation surfaces. R. López and M. I. Munteanu [9] constructed translation surfaces in $\mathrm{Sol}_{3}$ and investigated properties of minimal one.

In a different aspect, H. Liu [6] considered the translation surfaces with constant mean curvature in 3-dimensional Euclidean space and Lorentz-Minkowski space.

Recently, K. Seo [14] gave a classification of the translation hypersurfaces with constant mean curvature or constant Gauss-Kronecker curvature in space forms.

Related works on minimal translation surfaces of $\mathbb{E}^{3}$ are [ [6], [11], [17]].

Theorem 3.1 ( [10]). i) The only translation surfaces with constant Gauss curvature $K=0$ are cylindrical surfaces.

ii) There are no translation surfaces with constant Gauss curvature $K \neq 0$ if one of the generating curves is planar.

Definition 3.1. A homothetical (factorable) surface $M^{2}$ in 3-dimensional Euclidean space $\mathbb{E}^{3}$ is a surface that is a graph of a function

$$
z(u, v)=f(u) g(v)
$$

where $f: I \subset \mathbb{R} \rightarrow \mathbb{R}$ and $g: J \subset \mathbb{R} \rightarrow \mathbb{R}$ are two smooth functions.

Theorem 3.2 ( [10]). Planes and helicoids are the only minimal homothetical surfaces in Euclidean space.

Accordingly, we define an extended surface in $\mathbb{E}^{3}$ using definitions as above and called it THA-type surface. 
Definition 3.2. A surface $M^{2}$ in 3-dimensional Euclidean space $\mathbb{E}^{3}$ is a THA-surface if it can be parameterized by

$$
r(s, t)=(s, t, A f(s+a t) g(t)+B(f(s+a t)+g(t))),
$$

where $A$ and $B$ are non-zero real numbers.

Remark 3.1. i) If $A \neq 0$ and $B=0$ in (3.1), then $M^{2}$ is a affine factorable (homothetical) surface.

ii) If $A=0$ and $B \neq 0$ in (3.1), then $M^{2}$ is a affine translation surface.

Theorem $3.3([7])$. Let $r(x, y)=(x, y, z(x, y)=f(x)+g(a x+y))$ be a minimal affine translation surface. Then either $z(u, v)$ is linear or can be written as

$$
z(u, v)=\frac{1}{c} \log \frac{\cos \left(c \sqrt{1+a^{2}} x\right)}{\cos [c(a x+y)]} .
$$

Remark 3.2. If $a=0$, the minimal affine translation surface given by (3.2) is the classical Scherk surface.

Definition 3.3 ( [7]). The minimal affine translation surface (3.2) is called generalized Scherk surface or affine Scherk surface in Euclidean 3 - space.

Let $M^{2}$ be a THA-surface in $\mathbb{E}^{3}$ parameterized by (3.1). So

$$
r_{s}=\left(1,0, f_{s}(A g+B)\right), r_{t}=\left(0,1, g^{\prime}(A f+B)+a f_{t}(A g+B)\right) .
$$

The unit normal vector is given by

$$
\boldsymbol{N}=\frac{1}{W}\left(-f_{s}(A g+B),-\left(g^{\prime}(A f+B)+a f_{t}(A g+B)\right), 1\right),
$$

where $W^{2}=1+\left(f_{s}(A g+B)\right)^{2}+\left(g^{\prime}(A f+B)+a f_{t}(A g+B)\right)^{2}$.

The coefficients of the second fundamental form are given by

$$
\begin{gathered}
L=\frac{f_{s s}(A g+B)}{W}, M=\frac{a f_{s t}(A g+B)+A g^{\prime} f_{s}}{W}, \\
N=\frac{a^{2} f_{t t}(A g+B)+2 a A f_{t} g^{\prime}+g^{\prime \prime}(A f+B)}{W} .
\end{gathered}
$$

By a transformation

$$
\left\{\begin{array}{l}
x=s+a t \\
y=t
\end{array}\right.
$$


and $\frac{\partial(x, y)}{\partial(u, v)} \neq 0$.

From (3.3) and (3.1) we have

$$
r(x, y)=(x-a y, y, A f(x) g(y)+B(f(x)+g(y))) .
$$

The coefficients of the first fundamental form of $M^{2}$ are given by

$$
E=\frac{\alpha^{\prime 2} \gamma^{2}+A^{2}}{A^{2}}, \quad F=\frac{-a A^{2}+\alpha \gamma \alpha^{\prime} \gamma^{\prime}}{A^{2}}, G=\frac{\alpha^{2} \gamma^{\prime 2}+A^{2}\left(1+a^{2}\right)}{A^{2}}
$$

where $\alpha=A f+B$ and $\gamma=A g+B$.

The unit normal vector is given by

$$
\boldsymbol{N}=\frac{1}{W A}\left(-\gamma \alpha^{\prime},-\left(\alpha \gamma^{\prime}+a \gamma \alpha^{\prime}\right), 1\right),
$$

where $W^{2}=A^{-2}\left(\left(\gamma \alpha^{\prime}\right)^{2}+\left(\alpha \gamma^{\prime}+a \gamma \alpha^{\prime}\right)^{2}+A^{2}\right)$.

The coefficients of the second fundamental form are given by

$$
L=\frac{\gamma \alpha^{\prime \prime}}{A W}, \quad M=\frac{\alpha^{\prime} \gamma^{\prime}}{A W}, \quad N=\frac{\alpha \gamma^{\prime \prime}}{A W}
$$

4. THA-surfaces With ZERO GaUSSiAn CURVATURE IN $\mathbb{E}^{3}$

A surface in Euclidean 3-space parameterized by (3.4) has Gaussian curvature

$$
K=\frac{\alpha \gamma \alpha^{\prime \prime} \gamma^{\prime \prime}-\gamma^{\prime 2} \alpha^{2}}{A^{2} W^{4}}
$$

Hence that if $K=0$, then

$$
\alpha \gamma \alpha^{\prime \prime} \gamma^{\prime \prime}-\gamma^{\prime 2} \alpha^{2}=0
$$

We discuss the different cases according the functions $\alpha$ and $\gamma$.

The proof given in [12]. We can obtain the following:

Theorem 4.1 ( [12]). Let $M^{2}$ be a THA-surface in Euclidean 3 - space $\mathbb{E}^{3}$ with constant Gauss curvature $K$. Then $K=0$. Furthermore, the surface is plane or is a cylindrical surface over a plane curve or parameterized as (3.4), where 
i) either $f(x)=\lambda_{3} e^{k_{1} x}+\lambda_{4}$ and $g(y)=\lambda_{5} e^{k_{2} y}+\lambda_{6}$ or

ii) $f(x)=c_{3}\left((1-\lambda) k_{1} x+c_{1}\right)^{\frac{1}{1-\lambda}}+c_{4}$ and $g(y)=c_{5}\left(\left(\frac{\lambda-1}{\lambda}\right) k_{2} y+c_{2}\right)^{\frac{\lambda}{\lambda-1}}+c_{6}$.

\section{Minimal THA-surfaces in $\mathbb{E}^{3}$}

The expression of $H$ is

$$
H=\frac{\alpha \gamma^{\prime \prime}\left(\alpha^{\prime 2} \gamma^{2}+A^{2}\right)-2 \alpha^{\prime} \gamma^{\prime}\left(-a A^{2}+\alpha \gamma \alpha^{\prime} \gamma^{\prime}\right)+\gamma \alpha^{\prime \prime}\left(\alpha^{2} \gamma^{\prime 2}+A^{2}\left(1+a^{2}\right)\right)}{2 A^{3} W^{3}}
$$

Then $M^{2}$ is a minimal surface if and only if

$$
\alpha \gamma^{\prime \prime}\left(\alpha^{2} \gamma^{2}+A^{2}\right)-2 \alpha^{\prime} \gamma^{\prime}\left(-a A^{2}+\alpha \gamma \alpha^{\prime} \gamma^{\prime}\right)+\gamma \alpha^{\prime \prime}\left(\alpha^{2} \gamma^{\prime 2}+A^{2}\left(1+a^{2}\right)\right)=0
$$

We give examples of non-trivial minimal THA-surfaces by distinguishing some special cases:

Proposition 5.1. Let $M^{2}$ be a THA-surface in $\mathbb{E}^{3}$. If $M^{2}$ is minimal surface, then $M^{2}$ parameterized as (3.4), where

i) either $f(x)=x_{0}$ and $g(y)=\lambda_{1} y+\lambda_{2}$ or

ii) $g(y)=y_{0}$ and $f(x)=\lambda_{3} x+\lambda_{4}$ or

iii) $f(x)=\delta_{1} x+\delta_{2}$ and $g(y)=\delta_{3} \tan \left(\delta_{4} y+\delta_{5}\right)+\delta_{6}$ or

iv) $f(x)=\zeta_{1} \tan \left(\zeta_{2} x+\zeta_{3}\right)+\zeta_{3}$ and $g(y)=\xi_{1} y+\xi_{2}$.

6. THA-SURFACES SATISFYING $\Delta r_{i}=\lambda_{i} r_{i}$ IN $\mathbb{E}^{3}$

In this part we explore the classification of the THA-surfaces $M^{2}$ of $\mathbb{E}^{3}$ satisfying the condition

$$
\Delta r_{i}=\lambda_{i} r_{i}
$$

Lemma 6.1. The Laplacian $\Delta$ of $M^{2}$ can be expressed as follows:

$$
\Delta \varphi=\frac{-1}{W^{2}}\left[G \varphi_{x x}+E \varphi_{y y}-2 F \varphi_{x y}\right]+\frac{2 H}{W A}\left[Q(x, y) \varphi_{x}+P(x, y) \varphi_{y}\right]
$$

where

$$
Q(x, y)=a \alpha \gamma^{\prime}+\left(1+a^{2}\right) \gamma \alpha^{\prime}, \quad P(x, y)=a \gamma \alpha^{\prime}+\alpha \gamma^{\prime}
$$


Proof. From (2.1), we have

$$
\begin{aligned}
\Delta \varphi= & \frac{-1}{\sqrt{E G-F^{2}}}\left[\frac{\partial}{\partial x}\left(\frac{G \varphi_{x}-F \varphi_{y}}{\sqrt{E G-F^{2}}}\right)-\frac{\partial}{\partial y}\left(\frac{F \varphi_{x}-E \varphi_{y}}{\sqrt{E G-F^{2}}}\right)\right] \\
= & -\frac{1}{2 W^{4}}\left(2 W^{2}\left(G \varphi_{x x}-2 F \varphi_{x y}+E \varphi_{y y}\right)+\left(\left(G_{x}-F_{y}\right) 2 W^{2}-G^{2} E_{x}-E G G_{x}+\right.\right. \\
& \left.2 F G F_{x}+F G E_{y}+F E G_{y}-2 F^{2} F_{y}\right) \varphi_{x}+\left(\left(E_{y}-F_{x}\right) 2 W^{2}-E^{2} G_{y}-E G E_{y}+\right. \\
& \left.\left.2 F E F_{y}+F G E_{x}+F E G_{x}-2 F^{2} F_{x}\right) \varphi_{y}\right) .
\end{aligned}
$$

By (3.5) we find

$$
\begin{aligned}
& G_{x}=\frac{2 \alpha \gamma^{\prime} W M}{A}, \quad E_{x}=\frac{2 \gamma \alpha^{\prime} W L}{A}, \quad G_{y}=\frac{2 \alpha \gamma^{\prime} W N}{A} \\
& E_{y}=\frac{2 \gamma \alpha^{\prime} W M}{A}, \quad F_{x}=\frac{W\left(\alpha \gamma^{\prime} L+\gamma \alpha^{\prime} M\right)}{A}, \quad F_{y}=\frac{W\left(\alpha \gamma^{\prime} M+\gamma \alpha^{\prime} N\right)}{A} .
\end{aligned}
$$

Then

$$
\begin{aligned}
& 2\left(G_{x}-F_{y}\right) W^{2}-G^{2} E_{x}-E G G_{x}+F G\left(2 F_{x}+E_{y}\right)+F E G_{y}-2 F^{2} F_{y}=\mathcal{A}_{1} \\
& 2\left(E_{y}-F_{x}\right) W^{2}-E^{2} G_{y}-E G E_{y}+F E\left(2 F_{y}+G_{x}\right)+F G E_{x}-2 F^{2} F_{x}=\mathcal{A}_{2},
\end{aligned}
$$

where

$$
\mathcal{A}_{1}=-\frac{2 W H_{1}\left(a \alpha \gamma^{\prime}+\left(1+a^{2}\right) \gamma \alpha^{\prime}\right)}{A}, \quad \mathcal{A}_{2}=-\frac{2 W H_{1}\left(a \gamma \alpha^{\prime}+\alpha \gamma^{\prime}\right)}{A}
$$

and $H_{1}=E N+G L-2 F M$.

Applying (6.2) on the coordinate functions

$r_{1}(x, y)=x-a y, r_{2}(x, y)=y$ and $r_{3}(x, y)=A f(x) g(y)+B(f(x)+g(y))=A^{-1}\left(\alpha \gamma-B^{2}\right)$ of the position vector $r$ we find

$$
\left\{\begin{array}{l}
\Delta(x-a y)=\frac{2 H \gamma \alpha^{\prime}}{A W} \\
\Delta(y)=\frac{2 H\left(a \gamma \alpha^{\prime}+\alpha \gamma^{\prime}\right)}{A W} \\
\Delta\left(\frac{\alpha \gamma-B^{2}}{A}\right)=\frac{-2 H}{W}
\end{array}\right.
$$

By using (6.1) and (6.3) we have the following equations 


$$
\begin{aligned}
\frac{2 H \gamma \alpha^{\prime}}{A W} & =\lambda_{1}(x-a y) \\
\frac{2 H\left(a \gamma \alpha^{\prime}+\alpha \gamma^{\prime}\right)}{A W} & =\lambda_{2}(y) \\
\frac{-2 H}{W} & =\lambda_{3}\left(\frac{\alpha \gamma-B^{2}}{A}\right) .
\end{aligned}
$$

Therefore, the problem of classifying the affine translation surfaces $M^{2}$ satisfying (6.1) is reduced to the integration of this system of ordinary differential equations. Next we study it according to the constants $\lambda_{1}, \lambda_{2}, \lambda_{3}$.

Case 1. Let $\lambda_{3}=0$.

Then, the equation (6.6) gives rise to $H=0$, which means that the surfaces are minimal. We get also, by the equations (6.4) and (6.5), $\lambda_{1}=\lambda_{2}=0$.

Case 2. Let $\lambda_{3} \neq 0$. In this case we have four possibilities:

a) If $\lambda_{1}=0$ and $\lambda_{2}=0$ equations (6.4) and (6.5) imply that

$$
\begin{aligned}
\frac{2 H \gamma \alpha^{\prime}}{A W} & =0 \\
\frac{2 H\left(a \gamma \alpha^{\prime}+\alpha \gamma^{\prime}\right)}{A W} & =0 .
\end{aligned}
$$

a- 1) If $\alpha \gamma-B^{2}=0$. Then $\alpha^{\prime}=0$ and $\gamma^{\prime}=0$, which together with (5.1) leads to $H=0$.

a- 2) If $\alpha \gamma-B^{2} \neq 0$, equations (6.7) and (6.8) imply that $\alpha^{\prime}=0$ and $\gamma^{\prime}=0$. It then follows that the functions $\alpha$ and $\gamma$ are constants and that $H=0$. From (6.6) we obtain $\lambda_{3}=0$. So we get a contradiction.

b) If $\lambda_{1}=0$ and $\lambda_{2} \neq 0$, then $\alpha^{\prime}=0$, so $\alpha(x)=\lambda \in \mathbb{R}^{*}$. In this case the system (6.5) and (6.6) is reduced equivalently to

$$
\begin{aligned}
\frac{A^{2} \lambda^{2} \gamma^{\prime} \gamma^{\prime \prime}}{\left(A^{2}+\lambda^{2} \gamma^{\prime 2}\right)^{2}} & =\lambda_{2} y \\
\frac{-\lambda \gamma^{\prime \prime}}{W^{4}} & =\lambda_{3}\left(\lambda \gamma-B^{2}\right) .
\end{aligned}
$$

Equation (6.9) writes as 


$$
A^{2}\left(\frac{1}{A^{2}+\lambda^{2} \gamma^{\prime 2}}\right)_{y}=-2 \lambda_{2} y .
$$

A direct integration implies that there exist $c_{1} \in \mathbb{R}$ such that

$$
\frac{A^{2}}{A^{2}+\lambda^{2} \gamma^{\prime 2}}=-\lambda_{2} y^{2}+c_{1},
$$

where $0<-\lambda_{2} y^{2}+c_{1}<1$.

Multiplying equation (6.10) by $\gamma^{\prime}$ we get

$$
-\frac{A^{4} \lambda \gamma^{\prime} \gamma^{\prime \prime}}{\left(A^{2}+\lambda^{2} \gamma^{\prime 2}\right)^{2}}=\lambda_{3} \gamma^{\prime}\left(\lambda \gamma-B^{2}\right) .
$$

By integrating, we obtain that there exists a constant $c_{2}$ such that

$$
\frac{A^{4}}{A^{2}+\lambda^{2} \gamma^{\prime 2}}=\lambda_{3}\left(\lambda \gamma-B^{2}\right)^{2}+c_{2} .
$$

Comparing the equations (6.11) and (6.12) we get

$$
A^{2}\left(-\lambda_{2} y^{2}+c_{1}\right)=\lambda_{3}\left(\lambda \gamma-B^{2}\right)^{2}+c_{2}
$$

Then, we obtain

$$
\gamma=\frac{1}{\lambda}\left[B^{2}+\varepsilon \sqrt{\frac{-A^{2} \lambda_{2} y^{2}+c_{3}}{\lambda_{3}}}\right],
$$

where $c_{3} \in \mathbb{R}$ and $\varepsilon= \pm 1$.

c) If $\lambda_{2}=0$ and $\lambda_{1} \neq 0$. In this case the system (6.4), (6.5) and (6.6) is reduced equivalently to

$$
\begin{aligned}
\frac{2 H \gamma \alpha^{\prime}}{A W} & =\lambda_{1}(x-a y) \\
\frac{2 H\left(a \gamma \alpha^{\prime}+\alpha \gamma^{\prime}\right)}{A W} & =0 \\
\frac{-2 H}{W} & =\lambda_{3}\left(\frac{\alpha \gamma-B^{2}}{A}\right) .
\end{aligned}
$$

Then from (6.13) and (6.14) we have $\gamma \alpha^{\prime} \neq 0$, and $a \gamma \alpha^{\prime}+\alpha \gamma^{\prime}=0$. 
c-1) If $\gamma^{\prime}=0$, then $a \gamma \alpha^{\prime}+\alpha \gamma^{\prime}=0$ gives $a=0$, a contradiction.

c-2) If $\gamma^{\prime} \neq 0$, then $a \gamma \alpha^{\prime}+\alpha \gamma^{\prime}=0$ and it follows that there exists $c_{1} \in \mathbb{R}^{*}$ such that

$$
-\frac{1}{a} \frac{\gamma^{\prime}}{\gamma}=c_{1}=\frac{\alpha^{\prime}}{\alpha}
$$

Hence

$$
\gamma^{\prime}=-a c_{1} \gamma, \quad \alpha^{\prime}=c_{1} \alpha
$$

Then (6.15) writes as

$$
\begin{aligned}
\left(-\lambda_{3} A^{4} B^{2}\right) & +A^{4}\left(c_{1}^{2}+\lambda_{3}\right) \alpha \gamma-\left(2 \lambda_{3} A^{2} B^{2} c_{1}^{2}\right) \alpha^{2} \gamma^{2}+\left(2 \lambda_{3} A^{2} c_{1}^{2}\right) \alpha^{3} \gamma^{3} \\
& -\left(\lambda_{3} B^{2} c_{1}^{4}\right) \alpha^{4} \gamma^{4}+\left(\lambda_{3} c_{1}^{4}\right) \alpha^{5} \gamma^{5}=0
\end{aligned}
$$

For each fixed $x$, we can view this expression as a polynomial equation on $\gamma(y)$ and thus, all coefficients vanish. Then $\lambda_{3} A^{4} B^{2}=0$, a contradiction.

d) If $\lambda_{1} \neq 0$ and $\lambda_{2} \neq 0$ equations (6.4) and (6.6) imply that

$$
-A^{2} \lambda_{1}(x-a y)=\lambda_{3}\left(\alpha \gamma-B^{2}\right) \gamma \alpha^{\prime}
$$

Equations (6.5) and (6.6) imply that

$$
-A^{2} \lambda_{2} y=\lambda_{3}\left(\alpha \gamma-B^{2}\right)\left(a \gamma \alpha^{\prime}+\alpha \gamma^{\prime}\right)
$$

Then from (6.16) and (6.17) we have

$$
\left(\frac{\alpha}{\alpha^{\prime}}\right)\left(\frac{\gamma^{\prime}}{\gamma}\right)=\frac{\lambda_{2} y}{\lambda_{1}(x-a y)}-a .
$$

Differentiating (6.18) with respect to $x$, we get

$$
\left(\frac{\alpha}{\alpha^{\prime}}\right)_{x}\left(\frac{\gamma^{\prime}}{\gamma}\right)=-\frac{\lambda_{2} y}{\lambda_{1}(x-a y)^{2}} .
$$

Differentiating (6.18) with respect to $y$, we get 


$$
\left(\frac{\alpha}{\alpha^{\prime}}\right)\left(\frac{\gamma^{\prime}}{\gamma}\right)_{y}=\frac{\lambda_{2} x}{\lambda_{1}(x-a y)^{2}}
$$

From (6.19) and (6.20) we have

$$
\frac{-x\left(\frac{\alpha}{\alpha^{\prime}}\right)_{x}}{\left(\frac{\alpha}{\alpha^{\prime}}\right)}=\frac{y\left(\frac{\gamma^{\prime}}{\gamma}\right)_{y}}{\left(\frac{\gamma^{\prime}}{\gamma}\right)} .
$$

Since $\alpha$ and $\gamma$ are functions of two independent variables, we may write

$$
\frac{-x\left(\frac{\alpha}{\alpha^{\prime}}\right)_{x}}{\left(\frac{\alpha}{\alpha^{\prime}}\right)}=k=\frac{y\left(\frac{\gamma^{\prime}}{\gamma}\right)_{y}}{\left(\frac{\gamma^{\prime}}{\gamma}\right)}
$$

where $k \in \mathbb{R}$.

d-1) If $k=0$, then (6.22) follows

$$
\alpha=\delta_{1} \alpha^{\prime}, \quad \gamma^{\prime}=\delta_{2} \gamma
$$

where $\delta_{1}, \delta_{2} \in \mathbb{R}^{*}$.

Substituting this into (6.18), we get

$$
\lambda_{1}\left(\delta_{1} \delta_{2}+a\right) x-\left(a \lambda_{1}\left(\delta_{1} \delta_{2}+a\right)+\lambda_{2}\right) y=0 .
$$

Then $\lambda_{2}=0$, obtaining a contradiction.

d-2) If $k \neq 0$. Then (6.22), we obtain

$$
\frac{\alpha}{\alpha^{\prime}}=c_{1} x^{-k}, \quad \frac{\gamma^{\prime}}{\gamma}=c_{2} y^{k},
$$

where $c_{1}, c_{2} \in \mathbb{R}^{*}$.

Equation (6.18) writes as

$$
\lambda_{1} c_{1} c_{2} x^{-k} y^{k}(x-a y)=\lambda_{2} y-a \lambda_{1}(x-a y) .
$$


Differentiating with respect to $x$, we get $(1-k) x+(k+1) a y=0$, we conclude again a contradiction.

Consequently, we have:

Theorem 6.1. Let $M^{2}$ be a THA-surfaces given by (3.1) in $\mathbb{E}^{3}$. Then $M^{2}$ satisfies the equation $\Delta r_{i}=\lambda_{i} r_{i}(i=1,2,3)$ if and only if the following statement is true:

1) $M^{2}$ has zero mean curvature everywhere.

2) $M^{2}$ is parametrized as

$$
r(s, t)=\left(s, t, \frac{\varepsilon}{A} \sqrt{\frac{-A^{2} \lambda_{2} t^{2}+c_{3}}{\lambda_{3}}}\right) ; \quad c_{3} \in \mathbb{R}, \quad \varepsilon= \pm 1 .
$$

7. J-HARMONIC THA-surfaces IN $\mathbb{E}^{3}$

In this section we classify THA-surfaces in a Euclidean 3-space $\mathbb{E}^{3}$ satisfying the equation $\Delta^{J} r=0, J=I, I I$. A surface of in the three dimensional space is said to be $J$ - Harmonic if it satisfies the condition $\Delta^{J} r=0, J=I, I I$, where $\Delta^{J}$ denotes the Laplace operator with respect to the fundamental forms $I$ and $I I$.

7.1. I- Harmonic THA-surfaces in $\mathbb{E}^{3}$. Equation (6.3) writes as

$$
\Delta r(x, y)=\Delta^{I} r(x, y)=-2 H \mathbf{N}
$$

From (7.1), $\Delta^{I} r=0$ if and only if $H=0$.

Theorem 7.1. Let $r: M^{2} \rightarrow \mathbb{E}^{3}$ be an isometric immersion given by (3.4). Then $\Delta^{I} r=0$ if and only if $M^{2}$ has zero mean curvature.

7.2. II-Harmonic THA-surfaces in $\mathbb{E}^{3}$. In this section we are concerned with non-degenerate THA-surfaces $M^{2}$ without parabolic points satisfying the condition $\Delta^{I I} r=0$.

By a straightforward computation, the Laplacian $\Delta^{I I}$ of the second fundamental form $I I$ on $M^{2}$ with the help of (3.6) and (2.2) turns out to be

$$
\begin{aligned}
\Delta^{I I}= & -\frac{1}{A W D^{2}}\left(\alpha \gamma^{\prime \prime} \frac{\partial^{2}}{\partial x^{2}}+\gamma \alpha^{\prime \prime} \frac{\partial^{2}}{\partial y^{2}}-2 \alpha^{\prime} \gamma^{\prime} \frac{\partial^{2}}{\partial x \partial y}\right) \\
& -\frac{1}{2 D^{4}}\left(Q(x, y) \frac{\partial}{\partial x}+P(x, y) \frac{\partial}{\partial y}\right)
\end{aligned}
$$


where

$$
\begin{aligned}
& P(x, y)=\frac{1}{(A W)^{3}}\left(\alpha \alpha^{\prime} \alpha^{\prime \prime} \gamma \gamma^{\prime} \gamma^{\prime \prime \prime}-\alpha^{2} \alpha^{\prime \prime \prime} \gamma \gamma^{\prime \prime 2}-\alpha \alpha^{\prime} \alpha^{\prime \prime} \gamma \gamma^{\prime \prime 2}-2 \alpha^{3} \gamma^{2} \gamma^{\prime \prime}+3 \alpha \alpha^{\prime} \alpha^{\prime \prime} \gamma^{\prime \prime} \gamma^{2}\right), \\
& Q(x, y)=\frac{1}{(A W)^{3}}\left(\gamma \gamma^{\prime} \gamma^{\prime \prime} \alpha \alpha^{\prime} \alpha^{\prime \prime \prime}-\gamma^{2} \gamma^{\prime \prime \prime} \alpha \alpha^{\prime \prime 2}-\gamma \gamma^{\prime} \gamma^{\prime \prime} \alpha \alpha^{\prime \prime 2}-2 \gamma^{\prime 3} \alpha^{\prime 2} \alpha^{\prime \prime}+3 \gamma \gamma^{\prime} \gamma^{\prime \prime} \alpha^{\prime \prime} \alpha^{\prime 2}\right)
\end{aligned}
$$

and $D^{2}=L N-M^{2}$.

Accordingly, we get

$$
\begin{aligned}
\Delta^{I I} r(x, y) & =\left(\begin{array}{c}
\Delta^{I I}(x-a y) \\
\Delta^{I I}(y) \\
\Delta^{I I}\left(\frac{\alpha \gamma}{A}-\frac{B^{2}}{A}\right)
\end{array}\right) \\
& =-\frac{1}{2 D^{4}}\left(\begin{array}{c}
Q(x, y)-a P(x, y) \\
P(x, y) \\
4 W D^{4}+\frac{1}{A}\left(\alpha^{\prime} \gamma Q(x, y)+\alpha \gamma^{\prime} P(x, y)\right)
\end{array}\right) .
\end{aligned}
$$

$\Delta^{I I} r=0$, if and only if

$$
\left\{\begin{array}{l}
P(x, y)=0 \\
Q(x, y)=0 \\
W=0 .
\end{array}\right.
$$

From (7.2) we have $W=0$, which is a contradiction. Consequently, there are no THAsurfaces in $\mathbb{E}^{3}$ without parabolic points, satisfying the condition $\Delta^{I I} r=0$.

Finally, we mention the following classification:

Theorem 7.2. Let $M^{2}$ be a THA-surface given by (3.4), in $\mathbb{E}^{3}$. There are no THA-surface in $\mathbb{E}^{3}$ without parabolic points, satisfying the condition $\Delta^{I I} r=0$.

\section{References}

[1] M. Bekkar, B. Senoussi, Factorable surfaces in the three-dimensional Euclidean and Lorentzian spaces satisfying $\Delta r_{i}=\lambda_{i} r_{i}$, J. Geom. 103 (2012), 17 - 29.

[2] M. Bekkar, B. Senoussi, Translation surfaces in the 3-dimensional space satisfying $\Delta^{I I I} r_{i}=\mu_{i} r_{i}$, J. Geom. 103 (2012), 367-374.

[3] B.-Y. Chen, Total mean curvature and submanifolds of finite type, World Scientific, Singapore, (1984).

[4] F. Dillen, J. Pas, L. Verstraelen, On surfaces of finite type in Euclidean 3-space, Kodai Math. J. 13 (1990), 10-21. 
[5] A. Ferrandez, O.J. Garay, P. Lucas, On a certain class of conformally flat Euclidean hypersurfaces, Proc. of the Conf, in Global Analysis and Global Differential Geometry, Berlin. (1990).

[6] H. Liu, Translation surfaces with constant mean curvature in 3-dimensional spaces, J. Geom. 64 (1999), 141-149.

[7] H. Liu, Y. Yu, Affine translation surfaces in Euclidean 3-space, Proc. Japan Acad. 89 (2013), 111-113.

[8] R. López, Minimal translation surfaces in hyperbolic space, Beitr. Algebra Geom. 52 (2011), 105-112.

[9] R. López, M. I. Munteanu, Minimal translation surfaces in Sol 3 , J. Math. Soc. Japan, 64 (2012), 985-1003.

[10] R. López, M. Moruz, Translation and homothetical surfaces in Euclidean space with constant curvature, J. Korean Math. Soc. 52 (2015), 523-535.

[11] M. I. Munteanu, A. I. Nistor, Polynomial translation Weingarten surfaces in 3-dimensional Euclidean space, Proceedings of the VIII International Colloquium on Differential Geometry, World Scientific 2009, 316-320.

[12] B. Senoussi, M. Bekkar, Translation and homothetical TH-surfaces in the 3-dimensional Euclidean space $\mathbb{E}^{3}$ and Lorentzian-Minkowski space $\mathbb{E}_{1}^{3}$, Open J. Math. Sci. (2019), 74-83.

[13] B. Senoussi, M. Bekkar, Helicoidal surfaces with $\Delta^{J} r=A r$ in 3-dimensional Euclidean space, Stud. Univ. Babes-Bolyai Math. 60 (2015), no. 3, 437-448.

[14] K. Seo, Translation hypersurfaces with constant curvature in space forms, Osaka J. Math. 50 (2013), 631-641.

[15] B. O'Neill, Semi-Riemannian geometry with applications to relativity, Academic Press, Waltham (1983).

[16] T. Takahashi, Minimal immersions of Riemannian manifolds, J. Math. Soc. Japan. 18 (1966), 380-385.

[17] L. Verstraelen, J. Walrave, S. Yaprak, The minimal translation surfaces in Euclidean space, Soochow J. Math. 20 (1994), 77-82. 